\title{
Pragmatic Implementation of the Front-End of an N-bit/V ADC based on FPGA and FPAA
}

\author{
$1^{\text {st }}$ Salam Zayer \\ Institute of Automation and Infocommunication \\ University of Miskolc \\ Miskolc, Hungary \\ salaamzayer@gmail.com \\ $3^{\text {rd }}$ György Györök \\ Alba Regia Technical Faculty \\ Obuda University \\ Székesfehérvár, Hungar \\ gyorok.gyorgy@amk.uni-obuda.hu
}

\author{
$2^{\text {nd }}$ Marwah Muneer Al-bayati \\ Institute of Automation and Infocommunication \\ University of Miskolc \\ Miskolc, Hungary \\ marwamoner11@gmail.com \\ $4^{\text {th }}$ Ahmed Bouzid \\ Institute of Automation and Infocommunication \\ University of Miskolc \\ Miskolc, Hungary \\ qgebouzid@uni-miskolc.hu
}

\begin{abstract}
Reconfigurability has made it possible, among other benefits, to replace traditional discrete components with chips, whose internal components can be programmed in this case FPAAs (Field Programmable Analog Arrays). This paper presents a design and implementation of FPAA of the analog front end dedicated to a new ADC architecture called "Nbit/V". After validation of the algorithm in simulation, the experimentation results show that the obtained reconfigurable circuit can replace the traditional discrete components-based circuits.
\end{abstract}

\section{Keywords-ADC, FPAA, FPGA, Rapid Prototyping}

\section{NOMENCLATURE}

$\begin{array}{ll}\text { ADC } & \text { Analog-to-Digital Converter } \\ \text { ASIC } & \text { Application-Specific Integrated Circuit } \\ \text { CAB } & \text { Configurable Analog Block } \\ \text { FPAA } & \text { Field Programmable Analog Array } \\ \text { FPGA } & \text { Field Programmable Gate Array } \\ \text { IEEE } & \text { Institute of Electrical and Electronics Engineers } \\ \text { KSPS } & \text { Kilo Samples Per Second } \\ \text { OPAMP } & \text { Operational Amplifier } \\ \text { VLSB } & \text { Voltage Least Significant Bit } \\ \text { XADC } & \text { Xilinx Analog-to-Digital Converter } \\ \text { XSG } & \text { Xilinx System Generator }\end{array}$

\section{INTRODUCTION}

Alongside the development of the semiconductor technologies, manufacturers developed approaches to refresh their products with extra highlights on existing hardware. Programmable equipment whose sub-framework designs can be changed even after manufacture, falls under the classification of Reconfigurable System [1][2]. FPGAs (Field programmable gate arrays) are integrated circuits that, after being configured, can perform parallelly multiple digital processes. FPGAs have high programmable ability with low computational time. Field-programmable analog arrays (FPAAs) are coordinated reconfigurable circuits with an assortment of reconfigurable blocks associated through programmable nets allowing the design of analog circuits [3].
FPAA is a chip that can be programmed to execute various analog applications using the concept of configurable analog blocks (CAB). It is nowadays possible to perform a full frontend process on a single chip considering digital conditioning using FPGA thanks to the ease to use of high-level implementation tools such as XSG (Xilinx System Generator) [4]. FPAA can be an interesting choice for the analog designers, easy to use and can be incorporated into different analog designs, it forms an important model for analog designs and mixed systems without reservations [5]. [6] is discussed an application of combining FPGA and FPAA reconfiguration capabilities for IEEE 1451.5 for compliant smart sensor applications. In [7], the authors used FPAA and FPGA to implement a frontend interface for intelligent bioelectric signals acquisition system

In [8], the authors discussed the importance of the use of FPAA to implement mixed-signals processing systems emphasizing the efficiency in term of flexibility, hardware resources and power consumption, for instance, their use for very wide range frequencies applications. Nowadays, FPGAs support analog or mixed-signal applications with FPAA devices. Large-scale FPAAs can deal with ubiquitous analog or mixed-signal low-power sensor to process any similar device to the omnipresent implementation of the existing FPGA devices.

Huge scope FPAAs empower configurable and programmable calculation using both analog and digital methods. Old style advanced strategies are universal and powerful on account of boundary programmability and control-flow configurability [8]. In [9] the authors discussed the difference between FPAA and FPGA for implementation of signal processing (resumed in table I). Due to the limited availability of FPAA devices, it is difficult to establish a detailed study including all possible application areas of both approaches. In [10] the authors discussed the implementation of a speech detector in FPAA. The article shows that the power consumption of the FPAA is low. 
TABLE I. QUALITATIVE COMPARISON OF RECONFIGURABLE TECHNOLOGIES [9]

\begin{tabular}{lcc}
\hline Design Parameters & FPGA & FPAA \\
\hline Ease to implement signal processing applications & + & +++ \\
\hline Resources & +++ & - \\
\hline Development tools & +++ & +++ \\
\hline Power consumption & + & - \\
\hline Working frequency & +++ & + \\
\hline Efficiency & +++ & + \\
\hline
\end{tabular}

The authors in [11] designed a weather monitoring application using FPAA and FPGA then created the corresponding ASIC (Application-Specific Integrated Circuit) for weather monitoring application. Combining analog and digital reconfigurable circuits has already been investigated many times to enhance mixed signal based applications. For instance, the use of the couple FPAA-FPGA helped to automatically control the gain of an instrumentation amplifier for electrochemical applications [12]. Enhancing the resolution of an ADC has been treated in [13] where the authors proposed a combination of FPAA and FPGA. However, the system is based on discrete resistors to perform voltage division. Classical voltage dividers were often used for interfacing analog sensors with ADCs (Analog-to-Digital Converters) having low input ranges. The classical ADCs use resistors for voltage division. Thus, it has a limited duration of effectiveness. As resistors do age, even if some types are better than others, all resistors face this issue. For this reason, the classical ADC loses some accuracy after some years. Therefore, there is a need to create a new ADC architecture. This paper proposes an alternative solution to the work presented in [14] where the hybrid reconfigurability has been exploited to create a voltage attenuator to convert wider ranges voltages. The FPGA can solve the issue of the losing accuracy in the standard ADCs, as the software remains the same. For example, the 7 series Xilinx FPGAs provide an on-chip ADC called XADC (Xilinx Analog to Digital Converter), they are precise converters. Nevertheless, the need now is to design a system interfaced with the XADC, that can handle more than 1 peak-to-peak voltage.

\section{IMPLEMENTATION AND SIMULATION}

This paper introduces a system which can solve all the previously introduced issues using FPAA as front end of the FPGA. Figure 1 shows the whole model implemented in Simulink. Briefly, the working principle consists of splitting the input signal into 4 sub signals, each one is downshifted by $0,1,2$, and 3 Volts. At the same time the signal is compared with 1, 2, and 3 volts. These comparators outputs will help the digital processor (FPGA) to select which voltage range, the input voltage is situated then a controller will select the corresponding input to be converted by the embedded ADC. If we denote $A D C_{\text {out }}$ the output of the new ADC, the conversion process can be resumed in the following reasoning:

$$
A D C_{\text {out }}=X A D C_{\text {out }_{i}}+i
$$

where $X A D C_{\text {out }_{i}}$ is the output value of the embedded XADC, and $i$ is an index showing the voltage range to be converted of the voltage input $\left(V_{i n}\right)$ according to the following rule:

$$
i=\left\{\begin{array}{l}
0, V_{\text {in }} \in[0,1[ \\
1, V_{\text {in }} \in[1,2[ \\
2, V_{\text {in }} \in[2,3[ \\
3, V_{\text {in }} \in[3,4]
\end{array}\right.
$$

The output value of the XADC is the digitalized $i^{\text {th }}$ output of the FPAA $\left(F P A A_{\text {out }_{i}}\right)_{d}$ (selected by a combinatory circuit implemented on the FPGA) as following:

$$
X A D C_{\text {out }_{i}}=\left(\text { FPAA }_{\text {out }_{i}}\right)_{d}
$$

where $(X)_{d}$ is the digitalized value of an analog voltage $X$. The output voltage of the FPAA is none other than the voltage input downshifted by $i$ :

$$
\text { FPAA }_{\text {out }_{i}}=V_{\text {in }}-i
$$

The following formula resumes the whole process of the conversion using the new proposed ADC architecture:

$$
A D C_{\text {out }}=\left(V_{\text {in }}-i\right)_{d}+i
$$

Considering the specifications of the chosen FPGA and FPAA applied to the designed model, the results of the implementation can be seen in fig. 2. It can be observed that the results are interesting (Table II resumes the results), where 31 ADC clock cycles are required to perform a conversion, which means that the system is just consuming 5 more ADC clock cycles added to the 26 of the embedded XADC keeping the same resolution 12-bit, the same voltage resolution $244 \mu \mathrm{V}$ but with wider range $0-4 \mathrm{~V}$. The conversion rate is slightly lower than the original.

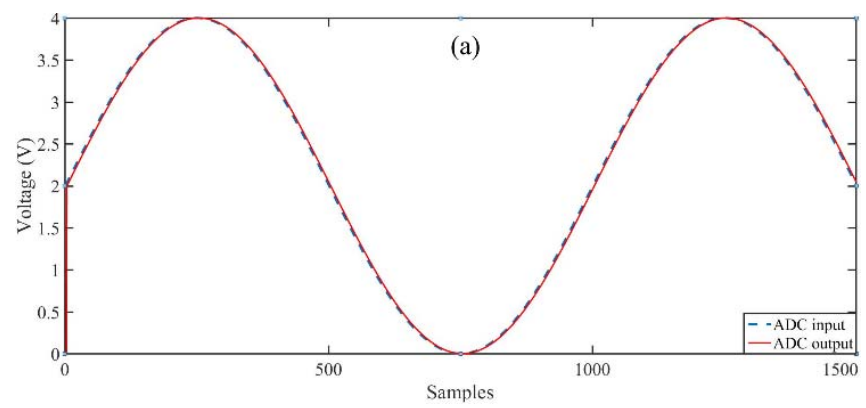

Fig. 1. Simulation results of the whole system considering two different waveforms [15]

TABLE II. PARAMETERS OF XADC AND THE PROPOSED 12-BIT/V ADC

\begin{tabular}{lll}
\hline Parameters & XADC & 12-bit/V ADC \\
\hline Conversion time (ADC clock cycles) & 26 & 31 \\
\hline Resolution (bits) & 12 & 12 \\
\hline $\mathrm{V}_{\mathrm{LSB}}(\mu \mathrm{V})$ & 244 & 244 \\
\hline Range $(\mathrm{V})$ & $0-1$ & $0-4$ \\
\hline Conversion rate (ksps) & 1000 & 838.7 \\
\hline
\end{tabular}




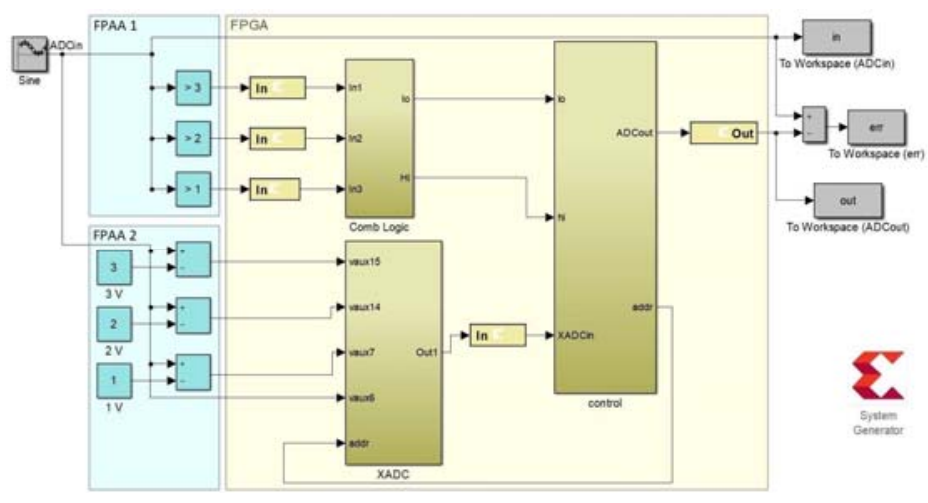

Fig. 2. Simulink model of the proposed system design [15]

The use of the first FPAA is to find the voltage range of the input, then pass the information (in 3 bits) to the FPGA. On the other side, the second FPAA shifts the input voltage into three different values and pass all of them to the FPGA as well. Table III and IV show the results of the calibration process for respectively FPAA1 and FPAA2. It can be observed that the output is unstable in a specific input range. For example, in comparator 1 , the output is not stable when the input voltage within the range of 2.93 and $2.99 \mathrm{~V}$. The same instability for other comparators but with different voltage ranges as shown in Table III.

TABLE III.

PARAMETERIZATION OF FPAA1

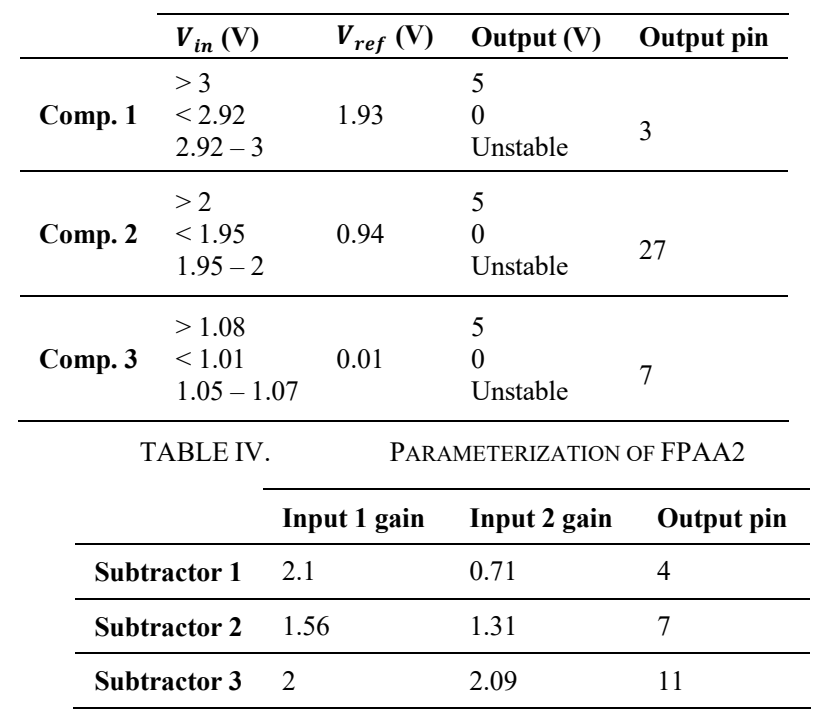

\section{RESUlTS AND DISCUSSION}

This paper presents an implementation and experimentation of the front end of a new ADC schematized in fig. 1 named N-bit/V ADC exploiting FPAA and FPGA hybridization. Figure 3 illustrates the circuit design implemented using Anadigm Software. The working principle as follows: The FPGA will receive 3-Bit clarifying the input range from FPAA1. Also, it will receive three different values of the shifted input from FPAA2. The circuit mainly contains three comparators, they compare the input to respectively $3 \mathrm{~V}$, $2 \mathrm{~V}$ and $1 \mathrm{~V}$. Mainly, FPAA2 contains three subtractors, each one shifts down the signal by a specific voltage. For better accuracy, an additional gain stage was needed before the subtractors. Figure 4 shows the equivalent electrical circuit of the front-end design. Comparing to what is expected from the mathematical model, some additional components have been added to the circuit since the components do not behave as in simulation. The calibration phase of the FPAA slows the prototyping phase, it is therefore time to consider an automated system for calibration. Figure 5 shows the results of the first configuration, while the yellow, green, blue, and magenta traces correspond to the input, the output of the comparator 1, 2, and 3, respectively. Table $\mathrm{V}$ shows the hardware and power resources of both FPAAs. It can be clearly seen that FPAA2 consumes more resources than FPAA1 as comparison does not consume as much energy and resources as subtraction and filtering.

\section{CONCLUSION}

Parameterization of FPAAs is not a straightforward matter since their behavior is not as deterministic as expected from simulation, a calibration phase is required. Initially, the whole reconfigurable analog part of the design was performed in a single FPAA chip, but a single net caused overconsumption of the resources. The next step will be to optimize the design to fit the configuration in a single FPAA chip. In the close future, the FPAA will be connected to the previously configured FPGA to test the whole ADC.

\section{ACKNOWLEDGMENT}

This research was supported by the European Union and the Hungarian State, co-financed by the European Regional Development Fund in the framework of the GINOP-2.3.4-152016-00004 project, aimed to promote the cooperation between the higher education and the industry.

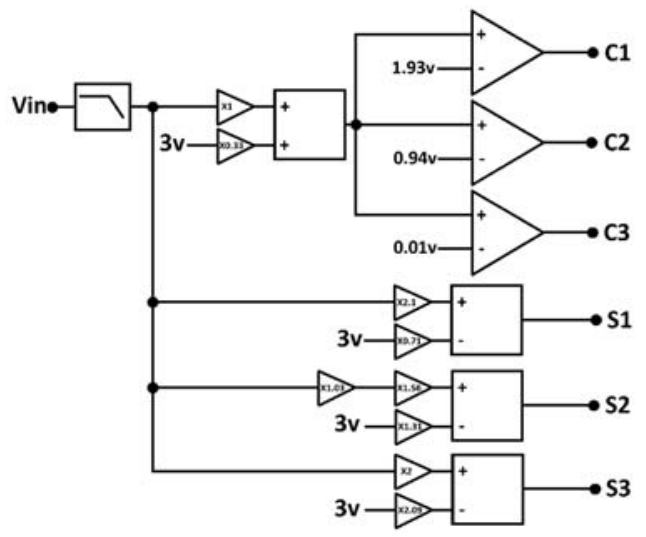

Fig. 3. The equivalent electrical circuit of both FPAAs design 

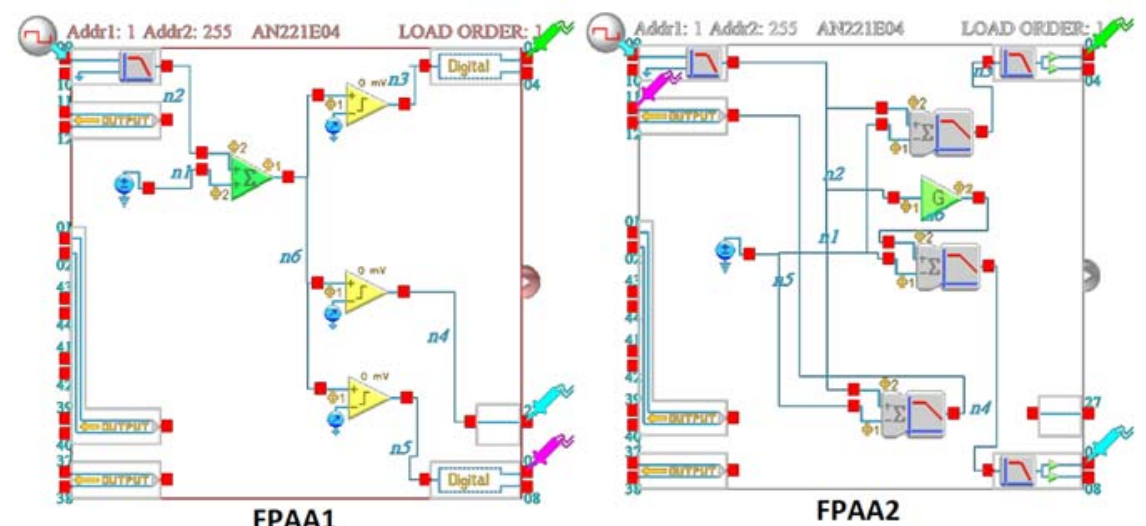

Fig. 4. Circuit of the reconfigurable analog front end implemented on FPAAs
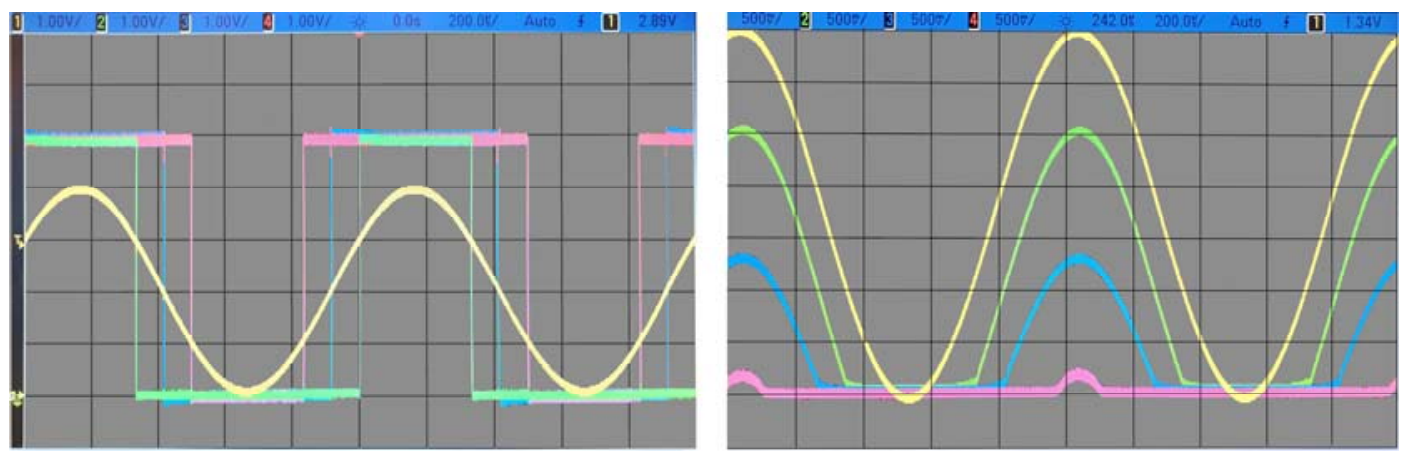

Fig. 5. Input and outputs of the FPAAs after configuration (FPAA1 on the left and FPAA2 on the right)

TABLE V. HARDWARE AND POWER RESOURCES OF BOTH FPAAS

\begin{tabular}{lll}
\hline Resources & Values of FPAA1 & Values of FPAA2 \\
\hline Chip & AN221E04 & AN221E04 \\
\hline OPAMPs & $1(12.5 \%)$ & $4(50 \%)$ \\
\hline Capacitors & $9(28.125 \%)$ & $14(43.75 \%)$ \\
\hline Comparators & $3(75 \%)$ & $0(0 \%)$ \\
\hline Inputs/outputs & $1 / 3$ & $1 / 3$ \\
\hline Power & $131 \pm 39 \mathrm{~mW}$ & $483 \pm 145 \mathrm{~mW}$ \\
\hline
\end{tabular}

\section{REFERENCES}

[1] Zhu, Jia, Michael Dexheimer, and Huanyu Cheng. "Reconfigurable systems for multifunctional electronics." npj Flexible Electronics 1.1 (2017): 1-13.

[2] Estrin, G.. "Reconfigurable Computer Origins: The UCLA Fixed-PlusVariable $(\mathrm{F}+\mathrm{V})$ Structure Computer." IEEE Ann. Hist. Comput. 24 (2002): 3-9.

[3] Baskaya, Ismail Faik. "Physical design automation for large scale field programmable analog arrays". PhD Dissertation. Georgia Institute of Technology, 2009.

[4] Meddah, Karim, et al. "FPGA-based system for heart rate monitoring." IET Circuits, Devices \& Systems 13.6 (2019): 771-782.

[5] Malcher, Andrzej, and Z. Kidoń. "Some properties of FPAA-based analog signal processing applications." IFAC Proceedings Volumes 42.1 (2009): 184-189.

[6] Morales, Diego P., et al. "Merging FPGA and FPAA reconfiguration capabilities for IEEE 1451.4 compliant smart sensor applications." 2007 3rd Southern Conference on Programmable Logic. IEEE, 2007.

[7] Mou, Pedro Antonio, et al. "Portable intelligent bioelectric signals acquisition system with an adaptive frontend implemented using FPGA and FPAA." World Congress on Medical Physics and Biomedical Engineering, September 7-12, 2009, Munich, Germany. Springer, Berlin, Heidelberg, 2009.

[8] J. Hasler, "Large-Scale Field-Programmable Analog Arrays," in Proceedings of the IEEE, vol. 108, no. 8, pp. 1283-1302, Aug. 2020

[9] Selow, Roberto, Heitor S. Lopes, and Carlos R. Erig Lima. "A comparison of FPGA and FPAA technologies for a signal processing application." 2009 International Conference on Field Programmable Logic and Applications. IEEE, 2009.

[10] Shah, Sahil, and Jennifer Hasler. "Low power speech detector on a FPAA." 2017 IEEE International Symposium on Circuits and Systems (ISCAS). IEEE, 2017.

[11] Vinayakamoorthy, Kumaresan, et al. "Design and implementation of ASIC for weather monitoring application using FPAA and FPGA." 2014 International Conference on Control, Instrumentation, Communication and Computational Technologies (ICCICCT). IEEE, 2014.

[12] Chen, Xuhai, and Min Du. "Automatic gain control for multi-channel electrochemical detection system combining FPGA and FPAA." 2012 5th International Conference on BioMedical Engineering and Informatics. IEEE, 2012.

[13] Morales, Diego P., et al. "Enhancing ADC resolution through Field Programmable Analog Array dynamic reconfiguration." 2008 International Conference on Field Programmable Logic and Applications. IEEE, 2008.

[14] Bouzid, Ahmed, and József Vásárhelyi. "Implementation and Experimentation of an Embedded Data Acquisition/Preprocessing System Based on a Hybrid Reconfigurable Hardware Accelerator for Inertial Measurements." IEEE Transactions on Industry Applications 56.2 (2020): 2012-2019.

[15] Bouzid, Ahmed, and József Vásárhelyi. "High Resolution Large Scale ADC. Case study of an N bit per Volt ADC Implemented using FPAA and FPGA Applied for Precision Altimetery." 2020 21st International Carpathian Control Conference (ICCC). IEEE, 2020. 